COMMISSION No. 42

\title{
CLOSE BINARY STARS (ETOILES DOUBLES SERREES)
}

\author{
Report of Meeting, 4 August 1988
}

PRESIDENT: J, SMGK

SECRETARY: B, J, Hrivnak

The business meeting of the commission was held in Room 103 of the Convention center on August 4 from 9:00-10:30, and was followed by a scientific meeting from 11:00-12:30.

The President extended a welcome to all present, and introduced a tentative agenda which was accepted. Dr. Bruce J. Hrivnak was then elected as Secretary for the meeting.

The President read the names of Commission members who had died during the previous three years: $M$. Fracassini, J. C. Kemp, and $M$. W. Ovenden and asked the attending members for a moment of silence to pay tribute to their memory.

The President announced that the organizing committee had recommended to the IAU Executive committee that the new Conmission President should be Robert H. Koch and the new Vice-President - Yoji Kondo. These recommendations were endorsed by the Cormission. Continuing members of the organizing Committee for 1988-91 are: E. Budding, A. M. Cherepashchuk, K.-C. Leung, J. Rahe, M. Rodono, J. Smak (as past President), and $G$. Shaviv. Vacancies on the Organizing Committee were then filled by the nomination and approval of A. Gimenez, R. W. Hilditch, R. F. Webbink, and A. Yamasaki, with the intention that a radio astronomer be added as soon as possible. The president expressed his appreciation to the Vice-president $R$, $H$. Koch and to the organizing comittee for their supoort and cooperation over the past three years. Concerning the new commission members, the President informed that all those who were nominated were approved by the organizing committee, and a final check is being performed to see that they have been elected as union members based upon nomination by their National Committees.

Tibor Herzeg discussed the status of the Bibliography and Program Notes, especially with regard to present difficult problems of printing and disseminating the pages. He raised for discussion the question whether the BPN should continue and, if so, whether he should continue as Editor. Following discussion, the continuation of both the Bibliography and Dr. Herzeg in his post was endorsed. The following resolution was adopted:

Recognizing the great value of the Bioliography and frogram notes to the work of IAU commission 42 , the commission expresses its gratitude to or. $T$. Herzeg and strongly encourages $h$ is continued work in editing this publication.

Alan H. Batten reported on the status of the Eighth Catalogue of Orbital Elements of spectroscopic Binary systems, noting some of the financial problems and difficult editorial decisions. A discussion ensued on the most appropriate means to publish the catalogue, its detailed format, and an editorial successor to Dr. Batten for future editions. The following resolution was adopted:

Recognizing the great value of the Eighth Catalogue of Orbital Elements of spectroscopic Binary systems to the work of IAU Commission 42 , the commission expresses its gratitude to Dr. A. H. Batten for his editorial and scholarly service. 
Robert H. Koch briefly addressed the subject of a new finding List of close Binary systems, noting a similarity with the problems raised by Dr. Batten. snortly a query will be mailed to users in order to gatner information regarding the desirability of a new edition. The following resolution was adopted:

Recognizing the great value of the finding List of Close Binary systems to the work of IAU Commission 42, the Commission expresses its gratitude to Dr. R. H. Koch and Dr, F, B. Wood.

R. H. Koch arew attention to two orinciples conveyed to the commission by the IAU President J, sanade. These are: (1) to note the circumstances of astronomers in disadvantaged locations and bring them into active ground-based and space work, and (2) to draw attention to coordinated observational programs of specific objects or classes of objects for specific intervals of time. He reported that the organizing committee was sympathetic to the principle of the first of these and drew attention to a Working Group concerned with Multi-Wavelength and Multi-Facility Programs.

A. H. Batten reported on the program of the laU Colloquium No. 107, Algols, to be held in Victoria following the General Assembly.

The commission recognized with appreciation the leadership by J. Smak as President during the past triennium.

During the scientific session the following papers were presented:

S. J. Schiller - The Hyades Binary HD 27130

M. B. K. Sarma - Distortion Wave Characteristics and Spot Modeling of the RS CVn Eclipsing Binary sv cam

J. V. Clausen - Recent Results from the Copennagen Binary Project

A. Gimenez and $A$. Claret - On the Internal structure of Main-sequence stars

G. Djurasevic - Analysis of Close Binaries by ADDlying Inverse-Problem Method

A. Cherepashonuk - Photometry of Ss 433 during 1979-87

G. Bakos - AW UMa. 
reported by $V$. Trimble

Alan Boss reviewed the four traditional mechanisms for forming binary systems -fragmentation, fission, capture, and separate nucleation, as well as the statistical properties of known binaries, which the formation mechanisms must account for. He concluded that we now understand both the data and the physical processes well enough to decide that the vast majority of field binaries must have originated from fragmentation. Additional fragmentation results were presented by Shinji Narita and Michel Bossi, the former suggesting that it may be very difficult to get succes sive stages of fragmentation (because the first will already leave gas clouds that are too centrally condensed to fragment further), and the latter reporting that a thermodynamic consideration of the process indicates the possibility of producing a bimodal distribution of mass ratios.

Fission simulations were reported by Harold Williams and Richard Durisen (in absentia). They concur that fission can make transient spiral arms, rings, and discs, but, so far, no binaries. Most of the mass, however, remains in a triaxial central bar, whose fate under further collapse is still to be determined.

Stellar encounters and captures are important in dense environments like cluster cores. Willy Benz reported that, when two-body encounters are close enough for something interesting to happen, disc formation and mergers are more likely that binary capture. Douglas Heggie presented results for three-body encounters and cap tures. What happens as a function of total energy, and the rate of formation of per manent bound pairs are now solved problems for clusters consisting of stars of a single mass. A clever way of generalizing to multi-mass systems is badly needed.

observers were under-represented relative to theorists, four of the originally scheduled speakers having developed assorted conflicts. Ramiro de la Reza discussed the properties of several pre-main-sequence binaries, including the isolated $\mathrm{T}$ Tau $\mathrm{V} 4046 \mathrm{Sgr}$. He noted that perhaps the most serious problem associated with such sys tems is understanding how they form, apparently in isolation from either gas or other young stars. Jean-Louis Halbwachs showed results of his analysis of samples of visual and spectroscopic binaries with main sequenc primaries. After allowance for selection effects, he concludes that the distribution of mass ratios in both samples is the same and consistent with random selection of components from a standard initial mass function. Discussion participants noted that it is possible to choose binary samples with different statistical properties, the BY Dra stars favoring large mass ratios and the $\mathrm{K}$ giants studied by $\mathrm{R}$. Griffin sma11 ones.

Finally, three talks addressed topics related to young binaries. Mikio Nagasawa has carried out a variety of three-dimensional, hydrodynamic simulations of tidal disruptions and related processes. David Bradstreet's investigation of the kinematics of $\mathrm{W}$ UMa stars finds that they have a relatively large average space motion of $66 \mathrm{~km} / \mathrm{s}$ and also a velocity dispersion characteristic of old disc populations. This favors a model for their formation in which systems gradually evolve in to contact from initial periods of 2-5 days. Tsevi Mazeh presented three samples of binaries of known ages in which the shortest orbit period that has not yet been circularized is correlated with age, from 10.7 days in $\mathrm{M} 67$ at $4-5 \times 10^{9} \mathrm{yr}$, through 5.7 days in clusters at $10^{8} \mathrm{yr}$, to 4 days in pre-MS systems with $10^{6} \mathrm{yr}$ ages. These num bers imply a dependence of circularization time or orbit period much less steep than the $16 / 3$ power law given by simple theory. 